\title{
Soft dielectric elastomer microactuator for robot locomotion
}

\author{
Farah Afiqa Mohd Ghazali ${ }^{1}$, Wong Yi Jie ${ }^{2}$, Mariatul Rawdhah Ahmad Fuaad ${ }^{3}$, \\ Mohamed Sultan Mohamed $\mathrm{Ali}^{4}$ \\ ${ }^{1,3,4}$ School of Electrical Engineering, Universiti Teknologi Malaysia Johor Bahru, Malaysia \\ ${ }^{2}$ i-Stone Systems Sdn Bhd 12-2, Jalan Persiaran Teknologi, Taman Teknologi Johor, Malaysia
}

\begin{tabular}{|c|c|}
\hline Article Info & ABSTRACT \\
\hline Article history: & This paper presents dielectric elastomer actuated robot locomotion and \\
\hline Received Dec 15, 2020 & $\begin{array}{l}\text { the development of a robotic model structure based using a dielectric } \\
\text { elastomer actuator. A pre-stretched dielectric elastomer actuator is fabricated }\end{array}$ \\
\hline Revised Mar 8, 2020 & onto acrylic frames to form single and multiple robotic crawler models. \\
\hline Accepted May 7, 2020 & $\begin{array}{l}\text { The crawler models demonstrate forwards motion upon application of high } \\
\text { voltage to the attached dielectric elastomer actuator. Characterizations }\end{array}$ \\
\hline Keywords: & $\begin{array}{l}\text { revealed that the fabricated multiple crawler models showed results over } \\
\text { the single crawler model in terms of locomotion potential. The maximum }\end{array}$ \\
\hline Dielectric elastomer & forward locomotion speed of the multiple crawler models is recorded as \\
\hline Electroactive polymer & $\begin{array}{l}1.2 \mathrm{~mm} / \mathrm{s} \text {. Nonetheless, precise results are highly attainable provided } \\
\text { a structured and coherent fabrication technique of the dielectric elastomer }\end{array}$ \\
\hline Microactuator & actuator is implemented. \\
\hline Robot locomotion & \\
\hline
\end{tabular}

This is an open access article under the CC BY-SA license.

\begin{abstract}
This paper presents dielectric elastomer actuated robot locomotion and the development of a robotic model structure based using a dielectric onto actic fram A The crawler models demonstrate forwards motion upon application of high voltage to the attached dielectric elastomer actuator. Characterizations revealed that the fabricated multiple crawler models showed results over the single crawler model in terms of locomotion potential. The maximum the multiple crawler models is recorded as actuator is implemented.
\end{abstract}

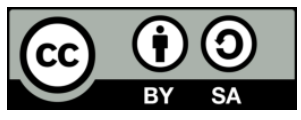

\section{Corresponding Author:}

Mohamed Sultan Mohamed Ali, School of Electrical Engineering, Universiti Teknologi Malaysia, 81310 UTM Johor Bahru Malaysia. Email: sultan_ali@fke.utm.my

\section{INTRODUCTION}

Soft type robots comprise of soft active materials which offer high compliance and adaptability compared to traditional hard robots [1]. Several soft actuations have been proposed and realized to demonstrate robotic locomotion such as hydrogels, pneumatic actuators and shape memory alloy actuators [2-8]. Each actuation method has distinguishable properties, distinct advantages and is applicable according to its capability in a specific situation. For instance, hydrogel has reliable biocompatibility, tunable mechanical and degradation features but its performance is limited due to slow response and relatively small force output [1]. Pneumatic actuators consist of chambers and are actuated by air pressure. Although it has been proven that they offer high flexibility, large displacement and high power-to-weight ratio, this type of actuator is relatively susceptible to rupture failures [9]. Shape memory alloy (SMA) deforms when stimulated by change in temperature and each has the ability to demonstrate large displacement [10-16]. Nonetheless, its constraints include a heat requirement and low efficiency [1]. Therefore, research on actuators have transitioned to soft actuators warranted that these actuators have the ability to address the issues faced by the previous actuators as well as provide better performance. Dielectric elastomer (DE) is defined as an electro active polymer and also known as a strain sensor that is triggered by the change of capacitance in a capacitor-like system. The working principle of DE is based on a system involving two compliant electrodes and an intermediate dielectric layer. Figure 1 illustrates the basic theory of DE actuator where the polymer material undergoes thickness reduction and area expansion. These two reactions are the immediate results of electrostatic force generated as voltage difference is applied to the electrodes. 


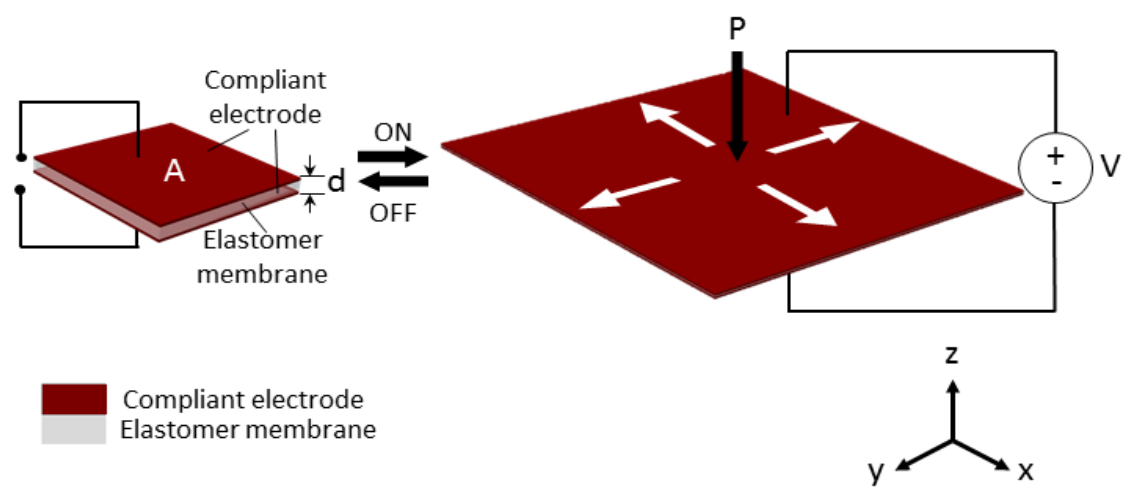

Figure 1. The DE actuate by electrostatic forces applied via compliant electrodes on the elastomer film

A study on an electro active polymer was conducted to investigate the energy density that may be potentially generated by each material and it was concluded that DE is reported to have ideal energy density. From the study, it was discovered that DE has a similar energy density of human muscles and is 8 times better than electromagnetic actuation [16, 17]. Dielectric elastomer actuation is also equated with a significantly good force-to-weight ratio and high strain potential [18-21]. Additionally, other methods have suggested that DE has the ability to reach up to $80 \%$ efficiency under constant charge operation mode [16]. Mimicking natural forms of movement has been established widely in the technology of artificial muscles. Dielectric elastomer actuator (DEA) has been a prime choice for integration into biomimetic devices due to the similarities with natural muscles. These intrinsic similarities make it easy to replicate natural systems and the multifunctionalities simplifies integrating sensing and load bearing elements into the design [22, 23].

DEAs are ideal for soft robotic application as it is known to significantly limit the total weight of the device as well as eliminate complex structures. Recently, a group reported an integration between a DEA and a hydraulically amplified self-healing electrostatic actuator which depends on the electrohydraulic mechanism to create a muscle-like movement [24]. Aside from that, several studies have been reported for successfully demonstrating bioinspired crawling motions [25-28]. One work presented robot locomotion actuated by polymer artificial muscle. The fabricated device was tested for its locomotion velocity, which was $0.245 \mathrm{~mm} / \mathrm{s}$ [25]. Another group reported a reconfigurable inchworm-inspired crawling robot operated by DEA. The device was designed to demonstrate vibrational and two-anchor crawling modes. Characterization of the device showed a locomotion speed of 0.9 body lengths per second and was able to carry $35 \%$ of its body weight [26]. Similarly, another elastomeric based inchworm locomotive was designed and reported [27]. The fabrication method comprised of a simple two-step manufacturing process and was characterized bas on actuator longevity and payload capacity [27]. Despite this, DEA based locomotive robots are prone to complex fabrication which limits its performance hence this issue has yet to be resolved.

In this paper, the integration between dielectric elastomer actuation and a locomotive robot application has been demonstrated. The characteristics of the dielectric elastomer actuated robotic device have been acquired based on the locomotion potential of different robotic models. The robot's locomotive speed was studied and analysed in order to characterize its potential capabilities

\section{PROPOSED METHOD}

\subsection{Design and working principle}

The working principle for DEA actuation essentially consists of a membrane sandwiched in between two flexibly compliant electrodes. Compressive electrostatic force is generated when high voltage is subjected to the electrodes and this causes the DEA to experience planar expansion and lateral compression. The change in electrostatic energy, $U$, per unit area, $A$, per unit displacement of the film in the direction of thickness, $z$, is known as the electrostatic force or Maxwell stress, $p$, which is expressed as:

$$
p=\left(\frac{1}{A}\right)\left(-\frac{d U}{d z}\right)
$$

Meanwhile, the electromechanical responses of compression due to expansion of the DEA is characterized as electrostatic pressure and expressed by the following equation:

$$
p=\varepsilon_{r} \varepsilon_{o} E^{2}=\varepsilon_{r} \varepsilon_{o}\left(\frac{V}{z}\right)^{2}
$$


where the permittivity of free space, relative permittivity or dielectric constant, voltage and membrane thickness are denoted as $\varepsilon_{0}, \varepsilon_{r}, V$ and $d$ respectively. This is followed by the elastic compressive strain, $s_{z}$, which is achieved by applying Hooke's law to an ideal elastomer. By incorporating an anisotropic Young's modulus $\mathrm{Y}$ independent of strain and a Poisson coefficient $v=0.5$, the compressive strain is denoted as

$$
S_{Z}=-\frac{p}{Y}=-\frac{\varepsilon_{0} \varepsilon_{r} E^{2}}{Y}=-\frac{\varepsilon_{0} \varepsilon_{r}}{Y}\left(\frac{V}{z}\right)^{2}
$$

In this work, the DEA is designed to activate locomotion for the robotic device. The robotic device measures $3 \mathrm{~cm}$ in width and $6 \mathrm{~cm}$ in height. VHB film was used as the dielectric elastomer film due to its high actuation stress and high strain properties. The circular acrylic frames were prepared via rapid prototyping process and used for pre-stretching the VHB films whereas rectangular acrylic frames were used for the locomotion itself. Carbon black powder was chosen as the conducting element due to its ability to display deformation alongside the flexible dielectric elastomer. Aside from that, it also offers relatively good electrical conductivity and high tolerance to strains. Bending joints were added to the robot device to allow actuation and forwards movement along a flat horizontal surface. $0.2 \mathrm{~mm}$ PVC sheets were used as the material for bending joints of the actuator.

In order to produce practical single crawler model design, thin wire was used instead of copper strips and PVC sheets are used to fabricate the legs for the device. The ground contacting point of the legs is cut into the sharp edge to maximize grip to the floor. The single crawler model design is shown in Figure 2(a) with flapper dimension of $4.0 \mathrm{~cm}$ in width and $5.0 \mathrm{~cm}$ in length. The multiple crawler model is designed by combining three of the single crawler models in the series which forms a three-segmented worm-like robot. Two weights are added to the front end and back end of the model to further enhance the grip to the floor. Without the weights, the actuator tends to flap upwards instead of forward which is not producing any thrusting movement. The model design is shown in Figure 2(b).

This robot model is inspired by mimicking earthworm locomotion. This model is able to produce forward movement without anti-reverse feature. Other than that, this robot model is able to perform in various kinds of surfaces as the friction force needed for anti-reverse mechanism is no longer a concern in this model. The working principle of this model combines the crawlers in series, which makes the model able to utilize the contracting motion of the deactivated segment (e.g. Crawler A) as the forward thrust for the segment (e.g. Crawler B) behind the deactivated crawler. Therefore, a steady forward thrust can be achieved by looping the sequence of voltage supply.

a)

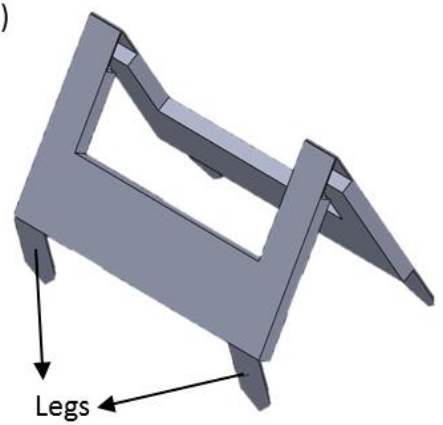

b)

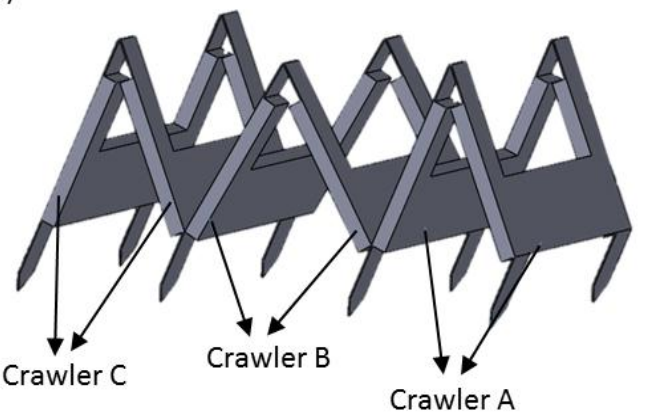

Figure 2. The crawler model design, (a) Single crawler, (b) Multiple crawler

\section{RESEARCH METHOD}

\subsection{Fabrication process}

Figure 3 has displayed the fabrication steps for the DEA prototype. The device was fabricated using low-cost, out-of-clean room fabrication technique using an acrylic frame and a pre-stretched DEA film. Firstly, an acrylic frame was prepared via rapid prototyping and used to pre-stretched VHB tape Figure 3(a). Next, the pre-stretched VHB tape is transferred onto the acrylic stiffener, followed by deposition of carbon black powder on the both sides of the VHB tape. Finally, strips of copper were used to attach the fabricated robot locomotion to the control circuit. Figure 3(b) shows the completed assembly of the locomotive prototype 
a)

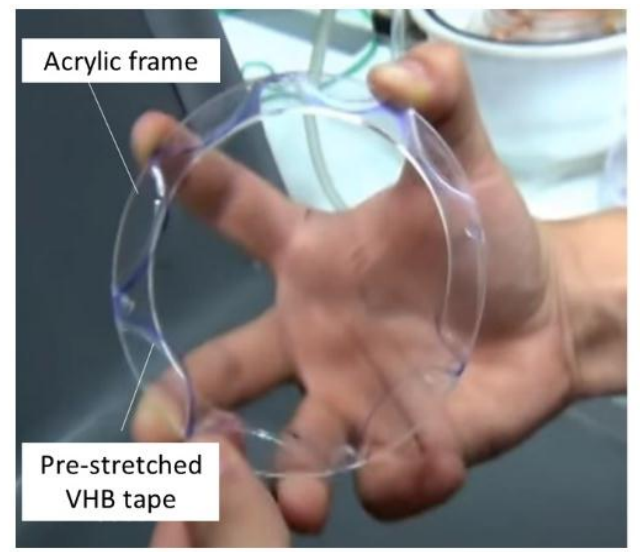

b) Stiffene

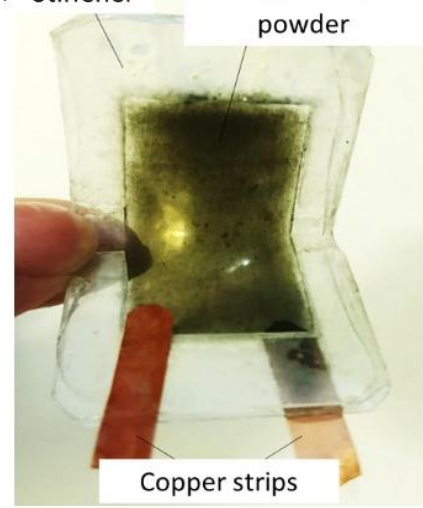

Figure 3. The fabrication steps for the DEA prototype, (a) Pre-stretched VHB tape on acrylic frame, (b) DEA prototype

\subsection{Experimental Setup}

The experimental setup for fabricated device characterization is displayed in Figure 4. A force gauge is used to measure the propelling force generated by robots. The force gauge is connected to a personal computer for graph plotting and data visualization as shown in Figure 4(a). Power supply is connected to a voltage amplifier module to step up $4.5 \mathrm{~V}$ to $5.0 \mathrm{kV}$. The experiment for single crawler model is set up as shown in Figure 4(b). Two aluminum bars are placed on both sides of the crawler as the conductors to the power supply. Thin aluminum foil strips $(10 \mu \mathrm{m})$ is used to keep the crawler and aluminum bar connected at the time the crawler is moving forward. The length of aluminum strips $(1.0 \mathrm{~cm})$ is short which is to keep the model as light as possible. The model is connected to power supply of $5 \mathrm{kV}$ with a frequency of $0.5 \mathrm{~Hz}$. The experiment for the multiple crawler model is set up as shown in Figure 4(c). The model is connected to a voltage supply of $5 \mathrm{kV}$.

The pulse is supplied to the model of sequence as shown in Figure 5. Then the process is repeated several times until supply is cut off. Aluminum bars is not implemented in this model as this experiment involved a more complicated voltage input procedure. Hence, long aluminum foil $(15.0 \mathrm{~cm})$ is used in this case which is adequate to remove the effects cause by stiffness of wires. The voltage pulse supplied to the model is shown in Figure 5 which is 0.5 second (s) from crawler A, B, to C. In both experiments, the locomotion performance is calculated by comparing the robot position after 25 seconds of actuation with its initial position. For measurement, the robot model is placed on a polyethylene plastic mat with $1 \mathrm{~cm} \times 1 \mathrm{~cm}$ grid pattern and actuated. The actuation of the device was analysed by image correlation technique to evaluate the robot locomotion.

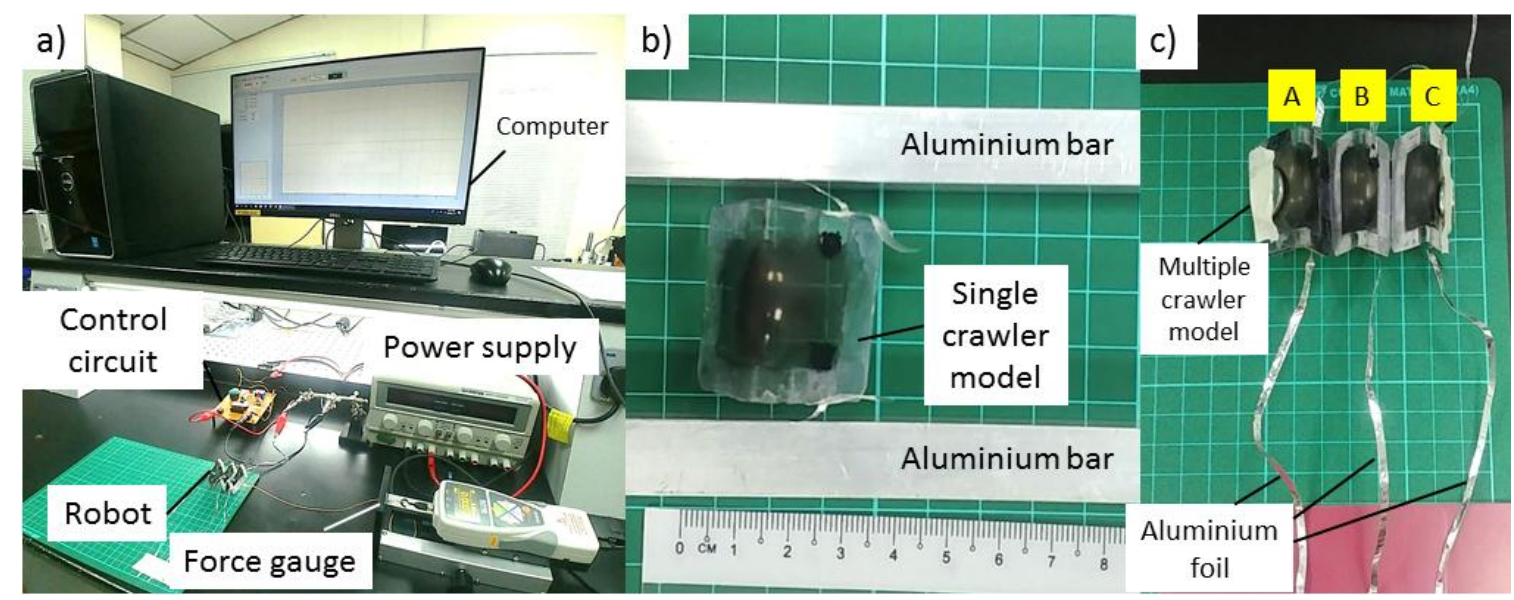

Figure 4. The experimental setup for fabricated device characterization, (a) Full experiment set up, (b) Experimental set up for single crawler model design, (c) Experimental set up for multiple crawler mode design 


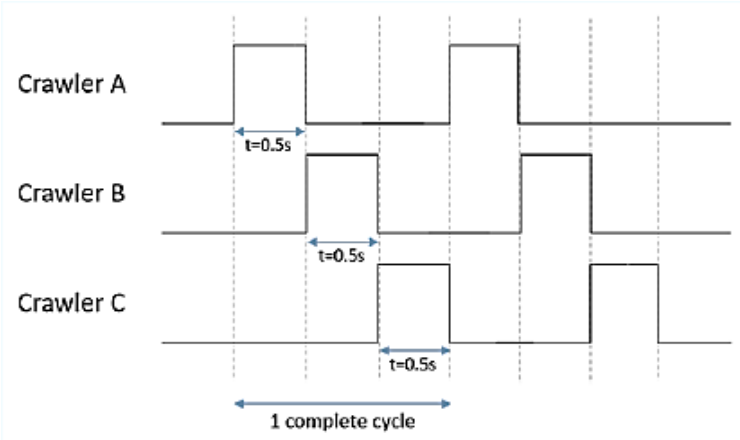

Figure 5. Voltage pulse supplied to multiple crawler model in sequence of A, B, C and repeat in loops

\section{RESULTS AND DISCUSSION}

In this section, the actuation of the fabricated crawler model is tested and characterized according to the speed of locomotion, the generated propelling force and the robot performance relationship against crawler activation period.

\subsection{Actuation Results}

The single crawler model has been tested on several surfaces including fabric, paper, rubber and wooden surfaces. For multiple crawler model, the robot design successfully mimicked a worm-like movement which is able to demonstrate a forward locomotion. Figure 6 depicts the locomotive movement demonstrated by the multiple crawler model whereas the experiment results of locomotion speed is shown in Table 1. From the experiment, the model moved a distance of $3.0 \mathrm{~cm}$ in 25 seconds. Furthermore, this robot model is also been tested by using different crawler activation period which is by $0.5,1.0,1.5,2.0$ and 2.5 second. Based on the best crawler activation period, $0.5 \mathrm{~s}$, propelling force generated by the model is measured from 0 to 5 second. The graph presenting the propelling force is generated as shown in Figure 7 .

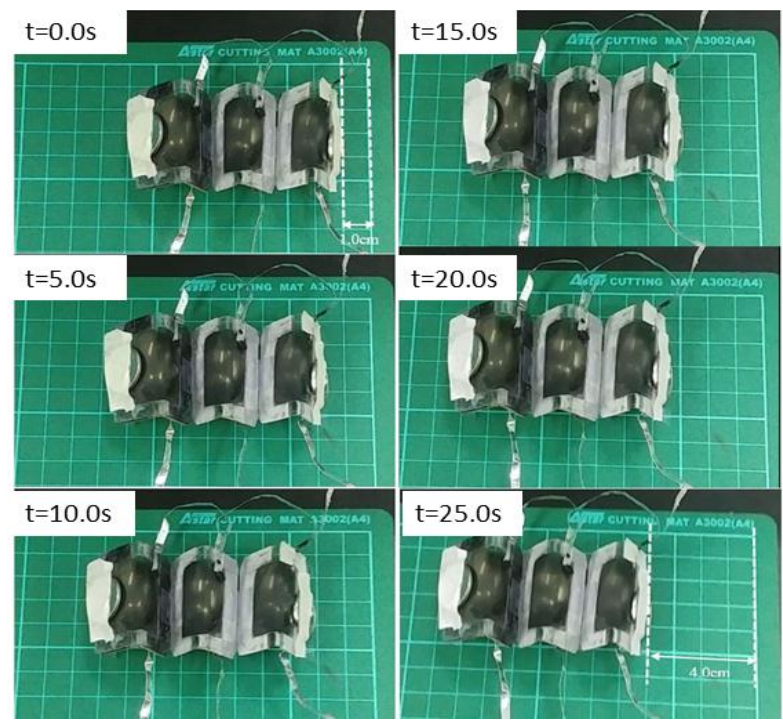

Figure 6. Crawling demonstration by multiple crawler model from 0.0 to 25.0 second

Table 1. Locomotion speed of the multiple crawler model

\begin{tabular}{ll}
\hline Crawler activation period $(\mathrm{s})$ & Locomotion speed $(\mathrm{mm} / \mathrm{s})$ \\
\hline 0.5 & 1.200 \\
1.0 & 0.700 \\
1.5 & 0.500 \\
2.0 & 0.333 \\
2.5 & 0.200 \\
\hline
\end{tabular}




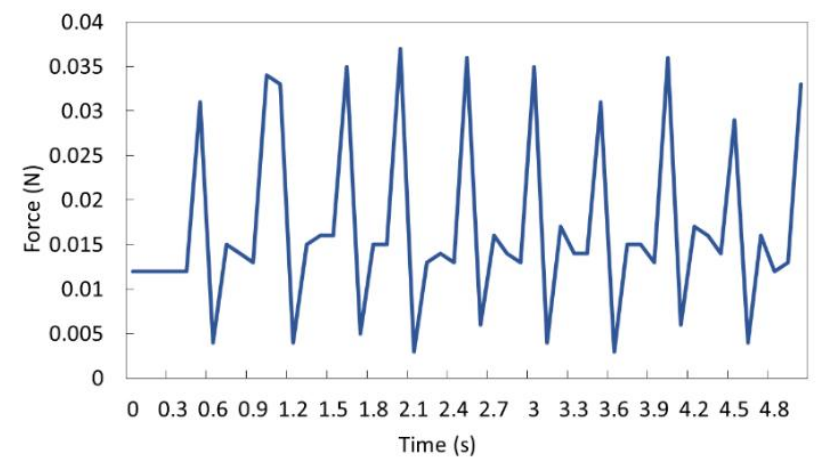

Figure 7. Propelling force by multiple crawler model

\subsection{Discussion}

One of the challenges to produce forward thrust by the single crawler model is to maintain the antireverse property between the contact of legs and floor surface. The weight of the robot is suspected to be too light to maintain the anti-reverse property. Besides, the weight of anti-reverse mechanism needs to be taken into consideration in robot designs so that the weight does not degrade the locomotion potential of robots. This kind of anti-reverse mechanism deserves further investigation. Based on the locomotion speed performance of the multiple crawler model, a graph is plotted in Figure 8 to help to visualize the effect of the crawler activation period.

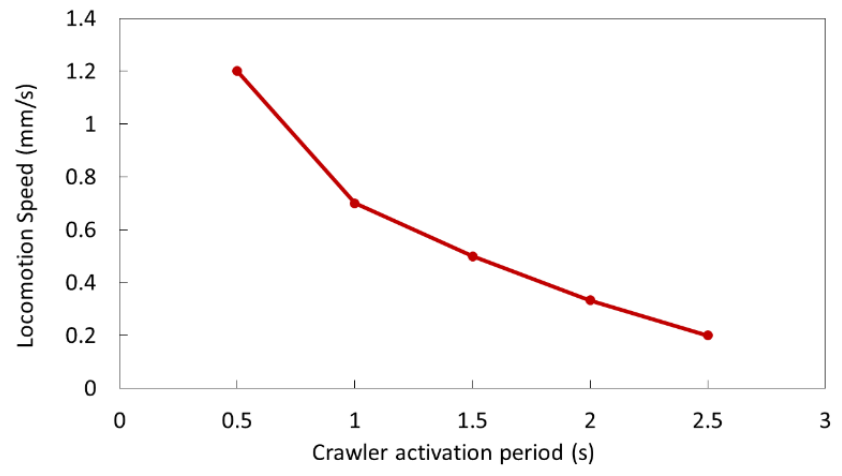

Figure 8. Robot performance relationship to change in crawler activation period

From the graph, it is gathered that the longer crawler activation period can cause a drawback of the robot locomotion speed. The resonance frequency of optimum locomotion performance of the robot model probably falls between 0 to 1 second of the crawler activation period. If the activation period is too short, the actuator will not be able to extend fully before the voltage input is switched to the next crawler segment, which will result in slow locomotion performance. While an activation period which is too long will result the actuators remain idle after being activated and causing drawback in performance as shown in the graph above.

\section{CONCLUSION}

This paper proposed an integration of the DE microactuator into a locomotive robot application. A single and multiple crawler model with two distinct configurations were designed and fabricated in order to demonstrate the locomotion movement of DE-microactuated robotic device. Locomotion movement configuration of the fabricate robots was observed whereas the performances was analysed based on the speed achieved by the models. From the characterization results, a movement speed of $1.2 \mathrm{~mm} / \mathrm{s}$ was achieved. In addition, the capability of the actuator is tested based on the difference in dimensions. The energy efficiency and effective locomotion are the main concern of this project when implementing DEA as a robot propeller. The biggest challenge faced by the model is to create an anti-reverse property 
between the contacts of the model with floor surfaces while keeping its weight as light as possible. Besides this, further improvements could be done regarding the fabrication process in order to improve the performance of the models. The fabrication process could be fully controlled by accurate measurement while thin wire can be used instead of aluminum foil which could further minimize the effect of wire stiffness. On the other hand, a wider research on materials, model design and control methods could be conducted as well to further improve the performance of DEAs.

\section{ACKNOWLEDGEMENTS}

This work has been supported by Contract Research Grant (CR 4C264) from Altech Industrial \& Engineering Supply Sdn Bhd. and Universiti Teknologi Malaysia (IIIG01M69)

\section{REFERENCES}

[1] U. Gupta, L. Qin, Y. Wang, H. Godaba, and J. Zhu, "Soft robots based on dielectric elastomer actuators: a review," Smart Materials and Structures, vol. 28, no. 10, pp. 103002, 2019.

[2] H. Qin, T. Zhang, N. Li, H-P. Cong, and S-H. Yu, "Anisotropic and self-healing hydrogels with multi-responsive actuating capability," Nature Communications, vol. 10, no. 1, pp. 1-11, 2019.

[3] J.-H. Lee, Y. S. Chung, and H. Rodrigue, "Long shape memory alloy tendon-based soft robotic actuators and implementation as a soft gripper," Scientific Reports, vol. 9, no. 1, pp. 1-12, 2019.

[4] M. Dahmardeh, M. S. M. Ali, T. Saleh, T. M. Hian, M. V. Moghaddam, A. Nojeh, K. Takahata, "High-power MEMS switch enabled by carbon-nanotube contact and shape-memory-alloy actuator," Physica Status Solidi A, vol. 210 , no. 4, pp. 631-638, 2013.

[5] A. AbuZaiter, M. Nafea, A. A. M. Faudzi, S. Kazi, and M. S. M. Ali, "Thermomechanical behavior of bulk NiTi shape-memory-alloy microactuators based on bimorph actuation," Microsystem Technologies, vol. 22, no. 8, pp. 2125-2131, 2016

[6] A. AbuZaiter, O. F. Hikmat, M. Nafea, and M. S. M. Ali, "Design and fabrication of a novel XY $\theta z$ monolithic micro-positioning stage driven by NiTi shape-memory-alloy actuators," Smart Materials and Structures, vol. 25, no. 10, pp. 105004, 2016.

[7] X. Qi, H. Shi, T. Pinto, and X. Tan, "A novel pneumatic soft snake robot using traveling-wave locomotion in constrained environments," IEEE Robotics and Automation Letters, vol. 5, no. 2, pp. 1610-1617, April 2020.

[8] H. Cui, Q. Zhao, Y. Wang, and X. Du, "Bioinspired actuators based on stimuli- responsive polymers," ChemistryAn Asian Journal, vol. 14, no. 14, pp. 2369-2387, 2019.

[9] D. Rus and M. T. Tolley, "Design, fabrication and control of soft robots," Nature, vol. 521, no. 7553, pp. 467-475, 2015.

[10] M. A. Zainal, S. Sahlan, and M. S. M. Ali, "Micromachined shape-memory-alloy microactuators and their application in biomedical devices," Micromachines, vol. 6, no. 7, pp. 879-901, 2015.

[11] A. AbuZaiter and M. S. M. Ali, "Analysis of thermomechanical behavior of shape-memory-alloy bimorph microactuator," 2014 5th International Conference on Intelligent Systems, Modelling and Simulation, pp. 390-393, 2014.

[12] A. AbuZaiter, E. Leen Ng, M. S. M. Ali, and S. Kazi, "Miniature parallel manipulator using TiNiCu shapememory-alloy microactuators," 2015 10th Asian Control Conference (ASCC), pp. 1-4, 2015.

[13] F. Sassa, Y. Al-Zain, T. Ginoza, S. Miyazaki, and H. Suzuki, "Miniaturized shape memory alloy pumps for stepping microfluidic transport," Sensors and Actuators B: Chemical, vol. 165, no. 1, pp. 157-163, 2012.

[14] H. Kato and K. Sasaki, "Transformation-induced plasticity as the origin of serrated flow in an NiTi shape memory alloy," International Journal of Plasticity, vol. 50, pp. 37-48, 2013.

[15] M. H. Elahinia, M. Hashemi, M. Tabesh, and S. B. Bhaduri, "Manufacturing and processing of NiTi implants: a review," Progress in Materials Science, vol. 57, no. 5, pp. 911-946, 2012.

[16] R. Pelrine, R. Kornbluh, J. Joseph, R. Heydt, Q. Pei, and S. Chiba, "High-field deformation of elastomeric dielectrics for actuators," Materials Science and Engineering: C, vol. 11, no. 2, pp. 89-100, 2000.

[17] N. H. Chuc, N. H. Lam Vuong, D. S. Kim, H. P. Moon, J. C. Koo, Y. Kwan Lee, Jae-Do N., and H. R. Choi, "Fabrication and control of rectilinear artificial muscle actuator," IEEE/ASME Transactions on Mechatronics, vol. 16, no. 1, pp. 167-176, Feb. 2011.

[18] R. Pelrine, R. Kornbluh, Q. Pei, and J. Joseph, "High-speed electrically actuated elastomers with strain greater than $100 \%$," Science, vol. 287, no. 5454, pp. 836-839, 2000.

[19] J.-S. Plante and S. Dubowsky, "On the performance mechanisms of dielectric elastomer actuators," Sensors and Actuators A: Physical, vol. 137, no. 1, pp. 96-109, 2007.

[20] S. M. Ha, W. Yuan, Q. Pei, R. Pelrine, and S. Stanford, "Interpenetrating networks of elastomers exhibiting 300\% electrically-induced area strain," Smart Materials and Structures, vol. 16, no. 2, pp. S280, 2007.

[21] F. A. M. Ghazali, C. K. Mah, A. AbuZaiter, P. S. Chee, and M. S. M. Ali, "Soft dielectric elastomer actuator micropump," Sensors and Actuators A: Physical, vol. 263, pp. 276-284, 2017.

[22] H. Godaba, J. Li, Y. Wang, and J. Zhu, "A soft jellyfish robot driven by a dielectric elastomer actuator," IEEE Robotics and Automation Letters, vol. 1, no. 2, pp. 624-631, July 2016.

[23] T. Li, G. Li, Y. Liang, T. Cheng, J. Dai, X. Yang, B. Liu, Z. Zeng, Z. Huang, Y. Luo, T. Xie, and W. Yang, "Fastmoving soft electronic fish," Science Advances, vol. 3, no. 4, pp. 1-7, 2017. 
[24] E. Acome, S. K. Mitchell, T. G. Morrissey, M. B. Emmett, C. Benjamin, M. King, M. Radakovitz, and C. Keplinger, "Hydraulically amplified self-healing electrostatic actuators with muscle-like performance," Science, vol. 359, no. 6371, pp. 61-65, 2018.

[25] Y. Yang, Y. A. Tse, Y. Zhang, Z. Kan, and M. Y. Wang, "A low-cost inchworm-inspired soft robot driven by supercoiled polymer artificial muscle," 2019 2nd IEEE International Conference on Soft Robotics (RoboSoft), pp. 161-166, 2019.

[26] C. Cao, R. S. Diteesawat, J. Rossiter and A. T. Conn, "A reconfigurable crawling robot driven by electroactive artificial muscle," 2019 2nd IEEE International Conference on Soft Robotics (RoboSoft), pp. 840-845, 2019.

[27] T. Duggan, L. Horowitz, A. Ulug, E. Baker, and K. Petersen, "Inchworm-inspired locomotion in untethered soft robots," 2019 2nd IEEE International Conference on Soft Robotics (RoboSoft), pp. 200-205, 2019.

[28] S. Chatterjee, R. Niiyama, and Y. Kawahara, "Design and development of a soft robotic earthworm with hydrostatic skeleton,"2017 IEEE International Conference on Robotics and Biomimetics (ROBIO), pp. 1-6, 2017.

\section{BIOGRAPHIES OF AUTHORS}

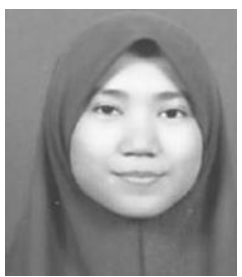

Farah Afiqa Mohd Ghazali received the B.Eng. degree in Mechanical Engineering from Universiti Teknologi Malaysia, Skudai, Malaysia in 2016. She is currently pursuing her Masters Degree in Electrical Engineering at Universiti Teknologi Malaysia. Her research interest is in the field of MEMS sensors and actuators.

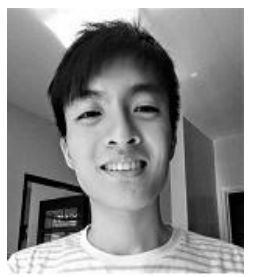

Wong Yi Jie received the B.Eng. degree in Electrical Engineering from Universiti Teknologi Malaysia, Skudai, Malaysia in 2019. Currently, he is working with i-Stone Systems Sdn Bhd, Senai, Johor, Malaysia.

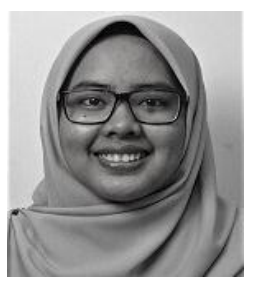

Mariatul Rawdhah Ahmad Fuaad received the Bachelor of Science in Science Instrumentation from Universiti Putra Malaysia, Serdang, Malaysia in 2018. She is currently pursuing her Masters Degree in Electrical Engineering at Universiti Teknologi Malaysia. Her research interest is in the field of MEMS actuators.

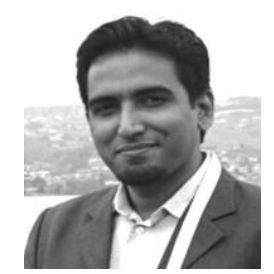

Mohamed Sultan Mohamed Ali received the B.Eng. and M.Eng. degrees in electrical engineering from Universiti Teknologi Malaysia, Skudai, Johor, Malaysia, in 2006 and 2008, respectively, and the Ph.D. degree in electrical and computer engineering from the Department of Electrical and Computer Engineering, The University of British Columbia, Vancouver, BC, Canada, in 2012. From 2001 to 2007, he held various engineering positions at Flextronics International Ltd. and Jabil Circuit, Inc. He is currently an Associate Professor in the Faculty of Electrical Engineering, Universiti Teknologi Malaysia. Dr. Mohamed Sultan serves as a consultant for Flextronics Malaysia in the failure analysis division and is actively involved in many industrial projects. He was awarded Chartered Engineer (C.Eng.) and Professional Engineer (P.Eng.) by United Kingdom Engineering Council and Board of Engineers Malaysia, respectively. Dr. Mohamed Sultan is also a Senior Member of IEEE (SMIEEE). Dr. Mohamed Sultan has published over 100 technical papers in MEMS and related fields. He has also served as the editor of 5 books in Mechatronics. His research interests are in the areas of MEMS, nanotechnology, and also in micro/nanofabrication technologies, including wireless microdevices, integration of microstructures, and microrobotics. 